\title{
DETERMINACIÓN DE ULTRATRAZAS DE CADMIO (II) POR TÉCNICAS VOLTAMÉTRICAS CON ELECTRODO DE CARBÓN VÍTREO MODIFICADO CON PELÍCULA DE BISMUTO
}

\author{
Hugo Ítalo Romero Bonilla ${ }^{\mathrm{a}^{*}, J}$ Jazmín Carolina Chiriboga Cabrera ${ }^{\mathrm{b}}$, \\ Alisson Paola Siguenza Balladares ${ }^{\mathrm{b}}$
}

\begin{abstract}
RESUMEN
El objetivo de este trabajo consistió en la determinación de ultratrazas de cadmio mediante la técnicas voltamétricas, modificando un electrodo de carbón vítreo con película de bismuto (BiFE). in situ con un potencial de pre-concentración $\mathrm{E}_{\text {prec }}=-1,2 \mathrm{~V}$ y un tiempo de deposición de $\mathrm{t}_{\text {prec }}=100 \mathrm{~s}$ en medio de acetato de sodio/ácido acético $0,1 \mathrm{M}(\mathrm{pH} 4,5)$ en ausencia de oxígeno, mediante voltamperometría de barrido lineal (LSV) con concentraciones en intervalos de $100-800 \mu \mathrm{g} . \mathrm{L}^{-1}$ y $2-10 \mathrm{mg} . \mathrm{L}^{-1}$ y voltamperometría de onda cuadrada (SWV) con concentraciones entre $50-800 \mu \mathrm{g} . \mathrm{L}^{-1}$. Se obtuvieron voltamogramas de potencial vs intensidad de la corriente y gráficos de concentración vs intensidad de la corriente, con linealidades de 0,997, 0,992 y 0,997 respectivamente. Se observó mejor sensibilidad en SWV observando picos más pronunciados tanto para cadmio y bismuto, ubicándose los potenciales de oxidación de cadmio para LSV en $-0,701 \mathrm{~V},-0,68 \mathrm{~V}$ y SWV en $-0,701 \mathrm{~V}$, respectivamente.
\end{abstract}

Palabras clave: cadmio, película de bismuto, carbón vítreo modificado, voltamperometría de barrido lineal, voltamperometría de onda cuadrada.

\section{DETERMINATION OF CADMIUM (II) ULTRATRACES BY VOLTAMMETRIC TECHNIQUES WITH BISMUTH FILM- MODIFIED VITREOUS CARBON ELECTRODE}

\begin{abstract}
The objective of this work was to determine cadmium ultratraces using voltammetric techniques, whit a vitreous carbon electrode modified with bismuth film (BiFE). in situ with $\mathrm{E}_{\text {prec }}=-1,2 \mathrm{~V}$ and a deposition time of $\mathrm{t}_{\text {prec }}=100 \mathrm{~s}$ in the medium of sodium acetate/acetic acid $0,1 \mathrm{M}(\mathrm{pH} 4,5)$ in the absence of oxygen, using linear scan voltammetry(LSV) whit concentrations of $100-800 \mu \mathrm{g} . \mathrm{L}^{-1}$ and $2-10 \mathrm{mg} . \mathrm{L}^{-1}$ and square wave voltammetry(SWV) whit concentrations of $50-800 \mu \mathrm{g} . \mathrm{L}^{-1}$. Were obtained voltamograms of potential vs intensity of the

a Grupo de Investigación Aplicaciones Electroanalíticas, Laboratorio de Electroanalítica y Bioenergía, Carrera Ingeniería Química, Universidad Técnica de Machala, Machala, El Oro, Ecuador, Código postal: 170517

*hromero@utmachala.edu.ec

b Semillero de Investigación Carrera de Ingeniería Química, Universidad Técnica de Machala, Machala, El Oro, Ecuador.
\end{abstract}


current and graphs of concentration vs intensity of the current, whit linearities of 0,997, 0,992 and 0,997 respectively. Was observed better sensitivity in SWV observing more pronounced peaks for both cadmium and bismuth, reaching the potential of oxidation of cadmium for LSV $-0,701 \mathrm{~V},-0,68 \mathrm{~V}$ and SWV in $-0,701 \mathrm{~V}$.

Key words: cadmium; bismuth film; vitreous carbon electrode; linear scan voltammetry; square wave voltammetry

\section{INTRODUCCIÓN}

El cadmio $\left(\mathrm{Cd}^{2+}\right)$ es un metal pesado muy tóxico que está presente en el medio ambiente debido a las actividades industriales realizadas por el hombre, entre sus usos están galvanoplastia, anticorrosivo, estabilizantes para plásticos, aleaciones y baterías, también en agroquímicos como los fertilizantes de fosfato ${ }^{1}$. Asimismo, trazas de este metal son causante de enfermedades como difusión renal, degeneración ósea, insuficiencia pulmonar, daño hepático e hipertensión ${ }^{2}$, dado el caso que el cadmio ingerido es significativamente mayor, en comparación al cadmio que ingresa al organismo mediante vía respiratoria ${ }^{3}$.

La espectroscopía de absorción atómica (AAS), espectroscopía de emisión de plasma acoplada inductivamente (ICPOES), espectrometría de plasma por inducción (ICP-MS) son técnicas ampliamente usadas para el análisis de metales pesados, pero son caras y poco adecuadas para la medición in-situ ${ }^{2}$. En sustitución a estas técnicas analíticas está la voltamperometría de redisolución anódica (ASV), que es una técnica muy útil y versátil para la determinación de iones metálicos traza. Posee óptima sensibilidad de lectura otorgada por un proceso de preconcentración del analito, el mismo que es depositado sobre la superficie del electrodo ${ }^{4}$, lo que hace posible la cuantificación de iones de metales pesados en ppb o en casos también en $\mathrm{ppt}^{5}$. Se ha demostrado que esta técnica muestra un notorio incremento en selectividad por algunos iones metálicos, cuando se implementa el uso de electrodos modificados químicamente ${ }^{6}$.

Como herramienta de detección inevitable de iones metálicos se encuentran los electrodos de película de mercurio (MFE), debido a su renovabilidad simple, alta sensibilidad, reproducibilidad para el electroanálisis voltamperométrico ${ }^{7}$. Sin embargo, la alta toxicidad del mercurio limita su aplicación llegando hacer restringido su uso como electrodo para efectuar análisis posteriores ${ }^{8}$. Es así que, el bismuto surge como una alternativa menos contaminante para ser utilizado como electrodo de película, el cual tiene facilidad de formar aleaciones de fusión con otros metales ${ }^{9}$. Adicionalmente, la toxicidad de sus sales son despreciables, por lo cual, no posee límites máximos permisibles por la OMS, es decir no presentan riesgo a la salud ${ }^{10}$. Asimismo, posee alta conductividad eléctrica, pretratamiento rápido, bajo costo, amplia disponibilidad y baja corriente de fondo, por lo que presenta ventajas en comparación a la película del mercurio ${ }^{11}$. 
Por otra parte, la concentración del electrolito soporte es importante ya que es clave primordial en la señal de ASV, pudiendo provocar interferencias al momento de la lectura ${ }^{12}$. El objetivo de este trabajo consistió en la determinación de cadmio mediante técnicas voltamétricas, con la modificación de un electrodo de carbón vítreo modificado con película de bismuto (BiFE).

\section{PARTE EXPERIMENTAL}

\section{Reactivos}

Se utilizó agua desionizada con una resistividad $(18,2 \mathrm{~m} \Omega . \mathrm{cm})$ obtenida de un sistema purificador de agua I y II para lavar y preparar las soluciones. Se preparó una solución tampón de acetato de sodio trihidratado más ácido acético $(99,99 \%)\left(\mathrm{NaCH}_{3} \mathrm{COO} .3 \mathrm{H}_{2} \mathrm{O}+\right.$ $\mathrm{HCH}_{3} \mathrm{COO}$ ) $0,1 \mathrm{M}$ con un $\mathrm{pH}$ de 4,5 como electrolito soporte a partir de reactivos de grado analítico. Se utilizó una solución estándar de $\mathrm{Cd}^{2+}$ con una concentración de $1000 \mathrm{mgL}^{-1}$. Todo el material de vidrio a utilizar se lavó previamente con una solución 1:1 de ácido nítrico (HNO3 64,9\%) enjuagando posteriormente con agua desionizada.

\section{Equipo}

Las mediciones voltamétricas de las soluciones se realizaron en un potenciostato PARSTAT MC Multi-Channel Potentiostat/Galvanostat marca Princeton Applied Research impulsado por software computarizado (VersaStudio) que garantiza un cómodo manejo y fácil acceso a las funciones del equipo. El cual se encontraba conectado a un sistema de tres electrodos. Se utilizó un electrodo de carbón vítreo como electrodo de trabajo de $3 \mathrm{~mm}$ de diámetro, un alambre de platino de $0,5 \mathrm{~mm}$ de diámetro como contraelectrodo, y como referencia un electrodo de $\mathrm{Ag} / \mathrm{AgCl}(3 \mathrm{M} \mathrm{KCl})$. Todas las mediciones se realizaron en una celda electroquímica de vidrio con una capacidad de $25 \mathrm{~mL}$ de solución electrolítica.

\section{Pre tratamiento de la superficie del electrodo de carbón vítreo}

El pre tratamiento del electrodo de carbón vítreo se lo realizó antes de ser modificado con la película de bismuto, el cual fue pulido sobre discos de tela marca BUEHLER con agua y polvo de alúmina (óxido de aluminio), utilizando primero la suspensión de 1,0 micrón y luego de 0,3 micrón.

Luego del pre tratamiento se realizó la activación electroquímica del electrodo mediante voltamperometría cíclica múltiple $(\mathrm{CV})$, con un barrido de potencial: $\mathrm{E}_{\mathrm{i}}=-1 \mathrm{~V} \mathrm{y} \mathrm{E}_{\mathrm{f}}=1 \mathrm{~V}$ a una velocidad de barrido $\mathrm{v}=20 \mathrm{mV} \cdot \mathrm{s}^{-1}$ y 50 Ciclos.

\section{Preparación de electrodo de carbón vítreo modificado con BiFE}

El estándar de bismuto se lo preparó a partir del nitrato de bismuto pentahidratado (Bi $\left.\left(\mathrm{NO}_{3}\right)_{3} .5 \mathrm{H}_{2} \mathrm{O}\right)$ pesando $0,1 \mathrm{~g}$ y aforándolo en un balón volumétrico de $100 \mathrm{ml}$ con el electrolito soporte. La modificación del electrodo se la realizó in-situ sobre la solución que contenía el analito, es decir, en la solución se encontraba iones $\mathrm{Cd}^{2+}$ e iones $\mathrm{Bi}^{3+}$. Posteriormente se fijó la concentración de $3 \mathrm{mg} . \mathrm{L}-1$ de $\mathrm{Bi}^{3+}$ en cada una de las muestras. 


\section{Procedimiento}

La preparación de las muestras de $\mathrm{Cd}^{2+}$ se realizó en balones volumétricos de $25 \mathrm{ml}$ a partir del estándar de $\mathrm{Cd}^{2+}$ de $1000 \mathrm{mg} . \mathrm{L}^{-1}$, en cada balón se colocaron las concentraciones requeridas de $\mathrm{Cd}^{2+}$ y $3 \mathrm{mg} . \mathrm{L}^{-1}$ de estándar de Bi para aforar con la solución electrolítica de acetato de sodio/ácido acético $(0,1 \mathrm{M}, \mathrm{pH} 4,5)$. Las mediciones electroquímicas de las soluciones se realizaron en ausencia de oxígeno, purgando las muestras con gas nitrógeno por un tiempo de $300 \mathrm{~s}$.

La electrodeposición de Cd sobre electrodo de carbón vítreo modificado con película de bismuto in situ se realizó aplicando una cronoamperometría $\mathrm{E}_{\mathrm{prec}}=-1,2 \mathrm{~V}, \mathrm{t}_{\mathrm{prec}}=100 \mathrm{~s}$.

Se aplicó voltametría de barrido lineal (LSV) a muestras con concentraciones de 100, 200, 400, 600 y $800 \mu 1 . \mathrm{L}^{-1}$ de $\mathrm{Cd}$ a potenciales de $-1,2 \mathrm{~V}$ a $0,2 \mathrm{~V}$ a una velocidad de barrido de $0,02 \mathrm{~V} \cdot \mathrm{s}^{-1}$ y muestras con concentraciones de 2,4,6,8 y $10 \mathrm{mg} \cdot \mathrm{L}^{-1}$ de $\mathrm{Cd}$ a las mismas condiciones.

Posteriormente se aplicó voltametría de onda cuadrada (SWV) a muestras con concentraciones de $50,100,200,400,600$ y $800 \mu \mathrm{g} . \mathrm{L}^{-1}$ a potenciales de $-1,1 \mathrm{~V}$ a $0,1 \mathrm{~V}$, altura de pulso $25 \mathrm{mV}$ y una frecuencia de $75 \mathrm{~Hz}$. Se realizaron cuatro réplicas de las soluciones.

\section{RESULTADOS Y DISCUSIÓN}

\section{Optimización de las condiciones analíticas}

Para obtener las mejores respuestas en la determinación de cadmio se utilizó el buffer de acetato de sodio/ácido acético $0,1 \mathrm{M}(\mathrm{pH} 4,5) 13$.

\section{Caracterización inicial de BiFE}

El comportamiento redox de BiFE se examinó mediante voltametría cíclica, en electrolito soporte de acetato de sodio/ácido acético $0,1 \mathrm{M}(\mathrm{pH} 4,5)$ con una concentración de $3 \mathrm{mg} . \mathrm{L}^{-1}$, en un rango de potencial de $-1,2 \mathrm{~V}$ a $0,2 \mathrm{~V}$, obteniendo un pico de oxidación de $-0,175 \mathrm{~V}$ y pico de reducción de $-0,363 \mathrm{~V}$ (figura 1), siendo el óptimo para la determinación del cadmio. Para ello se utilizaron electrolitos de $\mathrm{pH}$ 4,5 para evitar una evolución excesiva del hidrógeno ya que esto puede dificultar el proceso de deposición ${ }^{14}$. Como el bismuto tiene una amplia ventana de potencial negativo, es importante mantener el electrodo en potenciales más negativos a este, para así evitar su oxidación. En este sentido, se mantuvo el electrodo a potenciales más negativos que $-0,3 \mathrm{~V}$ en el electrolito acetato $0,1 \mathrm{M}(\mathrm{pH} 4,5)$ para mantener la integridad del revestimiento de bismuto. ${ }^{14}$ 


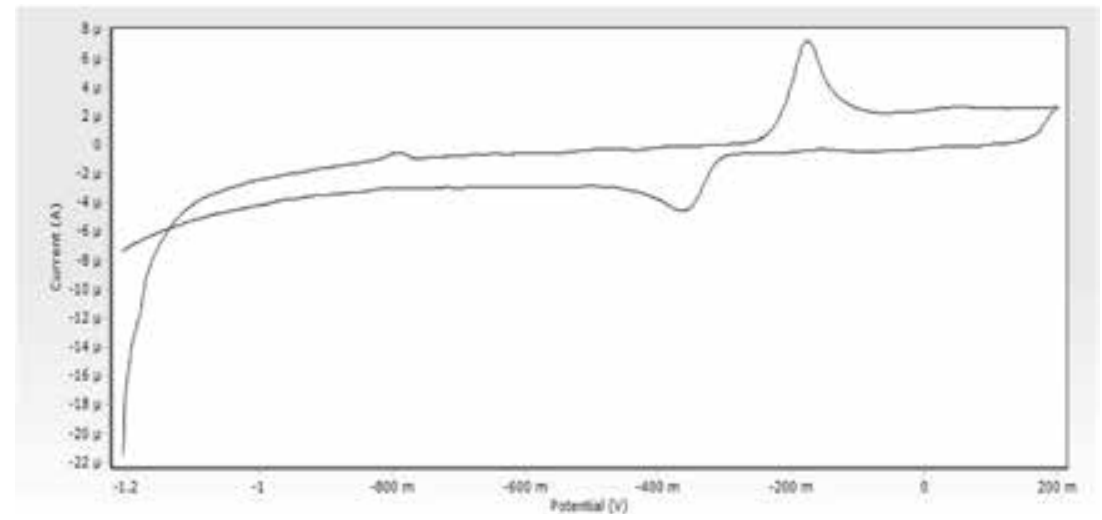

Figura 1. Voltamograma cíclico del comportamiento de bismuto en electrolito soporte de acetato $0,1 \mathrm{M}(\mathrm{pH} 4,5)$, a potenciales de $-1,2 \mathrm{~V}$ a $0,2 \mathrm{~V}$, pico de oxidación de $-0,175 \mathrm{~V}$ y pico de reducción de $-0,363 \mathrm{~V}$.

\section{Principio del método}

El método de ASV consiste principalmente en la deposición del cadmio $\left(\mathrm{Cd}^{2+} \mathrm{a} \mathrm{Cd}^{0}\right)$ sobre la superficie del electrodo de carbón vítreo. Luego de esto, se procedió a la etapa de redisolución, donde el cadmio se oxidó a su ión más estable. El cadmio con la aplicación del barrido lineal de potencial vuelve en forma iónica a la solución dando como resultado un incremento de corriente. A medida que se aumenta la concentración aumentará la corriente, por esto la corriente es proporcional a la concentración del cadmio, lo que nos permite cuantificar y así obtener datos de calibración para poder determinar la concentración del analito $\left(\mathrm{Cd}^{2+}\right)$ en muestras reales ${ }^{13}$.

\section{Comportamiento voltamétrico de $\mathrm{Cd}^{2+}$}

El comportamiento voltamétrico de cadmio con película de bismuto (BiFE) para el análisis ASV se realizó en muestras sintéticas por adición de solución estándar que contenían iones metálicos cadmio $\left(\mathrm{Cd}^{2+}\right)$ y bismuto $\left(\mathrm{Bi}^{3+}\right)$. La figura 2 (a y b) muestra una respuesta voltamétrica de barrido lineal, la cual se obtuvo en BiFE en una solución electrolítica de acetato de sodio/ácido acético $0,1 \mathrm{M}(\mathrm{pH} 4,5)$. En este caso al aplicar un potencial característico inicial de $-1,2 \mathrm{~V}$ y final de $0,2 \mathrm{~V}$ se obtiene como respuesta la corriente máxima como se observa en los voltamogramas a concentraciones de 100-800 $\mu \mathrm{g} . \mathrm{L}^{-1}$ en rango bajo (figura 2a) y, a concentraciones entre 2-10 mg. $\mathrm{L}^{-1}$ rango alto (figura $2 \mathrm{~b}$ ). En ambos casos se observan los picos anódicos definidos de cadmio, así como picos de bismuto co-depositado. Por otra parte, también se realizó la determinación de cadmio por voltametría de onda cuadrada a concentraciones de 50-800 $\mu \mathrm{g} . \mathrm{L}^{-1}$ como se observa en la figura 3 . En ella se puede observar una mejor sensibilidad, obteniéndose picos más definidos al aplicar un potencial inicial de $-1.1 \mathrm{~V}$ y final $0.1 \mathrm{~V}$ para lograr una mejor respuesta en corriente. 


\section{Validación del método}

Luego de la optimización de los parámetros de medición por el método de voltametría de barrido lineal se realizó la validación analítica del método, mediante la linealidad de las concentraciones por adición estándar de cadmio frente a la corriente máxima obtenida de la respuesta del ión cadmio. Las figuras 4 y 5 muestran la curva de calibración de cadmio, encontrándose una linealidad a concentraciones entre 100-800 $\mu \mathrm{g} . \mathrm{L}^{-1}$ (figura 4) y entre 2-10mg.L $\mathrm{L}^{-1}$ (figura 5), obteniendo una pendiente $6 \times 10^{-3} \mu \mathrm{A} / \mu \mathrm{g} . \mathrm{L}-1$ y un $\mathrm{R}^{2}$ de 0,997 para concentraciones de rango bajo y 4,326 $\mu \mathrm{A} . / \mathrm{mg} . \mathrm{L}^{-1}$ y un $\mathrm{R}^{2}$ de 0,992 para concentraciones de rango alto. Asimismo, la figura 6 muestra la curva de calibrado para el sistema de onda cuadrada, encontrándose la linealidad a concentraciones entre 50-800 $\mu \mathrm{g} . \mathrm{L}^{-1}$ con una pendiente de $0,1012 \mu \mathrm{A} / \mu \mathrm{g} . \mathrm{L}^{-1}$ y un $\mathrm{R}^{2}$ de 0,998 . Esto nos permite deternimar que sí se cumple con los parámetros establecidos según la FDA (Food and Drug Administration) (2001)15. Lo que nos permite cuantificar la concentración del analito $\left(\mathrm{Cd}^{2+}\right)$ de manera confiable en muestras reales.

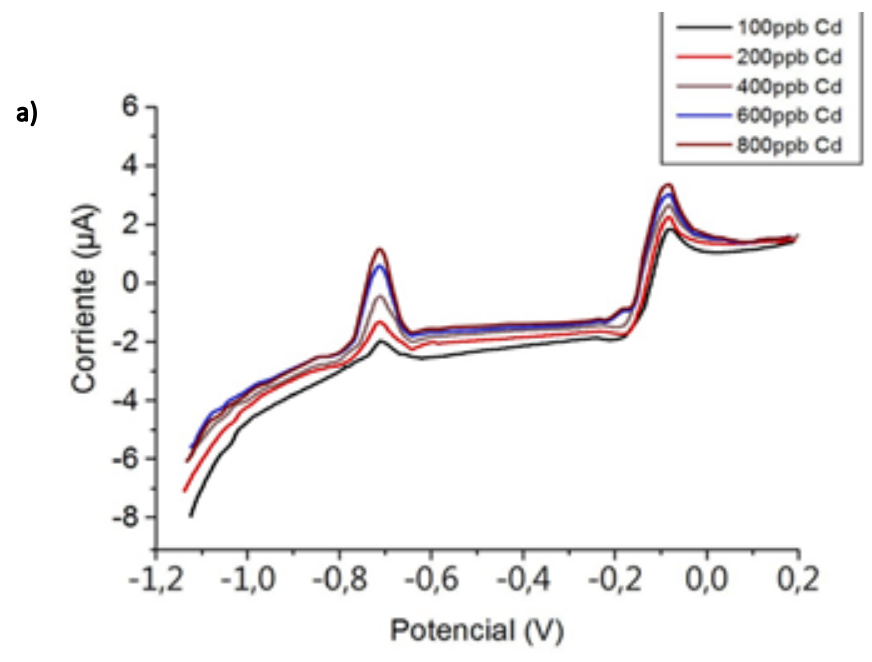


b)

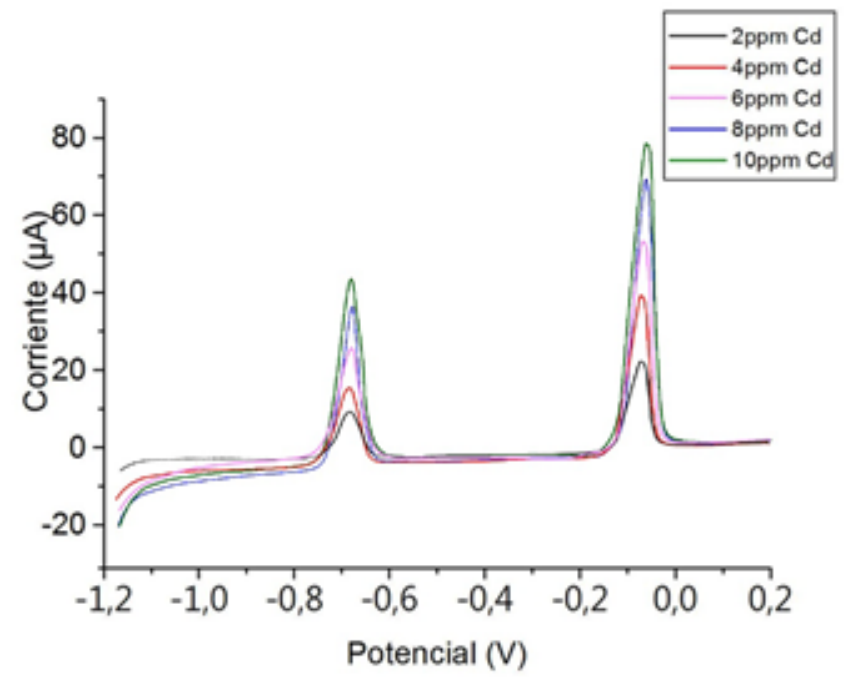

Fuente: Elaboración propia

Figura 2. Voltamograma de barrido lineal con electrodo de carbón vítreo modificado con

BiFE. en medio acetato de sodio/ácido acético $0,1 \mathrm{M} \mathrm{pH} 4,5$ a), concentración de $\mathrm{Cd}^{2+}$ $100,200,400,600,800 \mu \mathrm{g} \cdot \mathrm{L}^{-1}$ y b) 2,4,6,8,10 mg.L $\mathrm{L}^{-1}$, potencial de preconcentración $\mathrm{E}_{\text {prec }}=-1.2 \mathrm{~V}$, tiempo de deposición $\mathrm{t}_{\text {prec }}=100 \mathrm{~s}$, a potenciales de $-1,2 \mathrm{~V}$ a $0,2 \mathrm{~V}$ y velocidad de barrido de $0,02 \mathrm{~V}_{\mathrm{s}}{ }^{-1}$, Potencial de Oxidación del Cdmio $\mathrm{P}_{\text {oxi }}=-0,701 \mathrm{~V}$ y $-0,68 \mathrm{~V}$.

c)

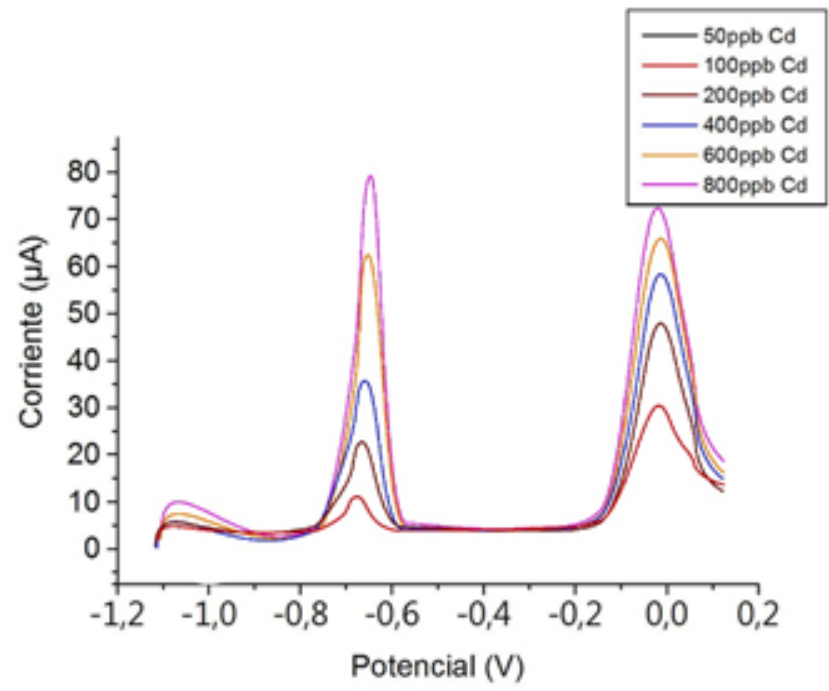

Fuente: Elaboración propia

Figura 3. VVoltamograma de Onda Cuadrada con electrodo de carbón vítreo modificado con BiFE. en medio acetato de sodio/ácido acético $0,1 \mathrm{M} \mathrm{pH} 4,5$, concentración de $\mathrm{Cd}^{2+}$ $50,100,200,400,600,800 \mu \mathrm{g} \cdot \mathrm{L}^{-1}$ potencial de preconcentración $\mathrm{E}_{\text {prec }}=-1,2 \mathrm{~V}$, tiempo de deposición $\mathrm{t}_{\text {prec }}=100 \mathrm{~s}$, a potenciales de $-1,1 \mathrm{~V}$ a $0,1 \mathrm{~V}$, altura de pulso de $25 \mathrm{mV}$ y una frecuencia de $75 \mathrm{~Hz}$, Potencial de Oxidación del Cdmio $\mathrm{P}_{\text {oxi }}=-0,701 \mathrm{~V}$. 


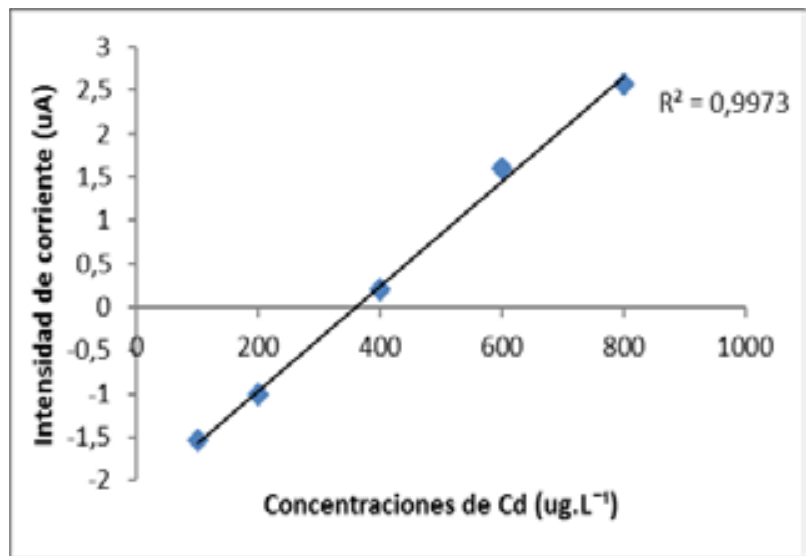

Fuente: Elaboración propia

Figura 4. Curva de calibración de cadmio por adición de soluciones estándar de 100, 200, 400,600 y $800 \mu \mathrm{g} . \mathrm{L}^{-1}$

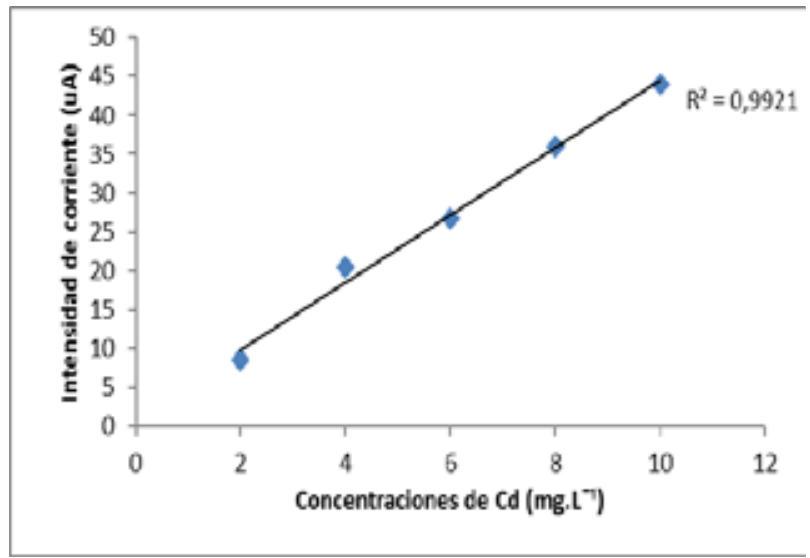

Fuente: Elaboración propia

Figura 5. Curva de calibración de cadmio por adición de soluciones estándar de 2, 4, 6, $8,10 \mathrm{mg} . \mathrm{L}^{-1}$ 


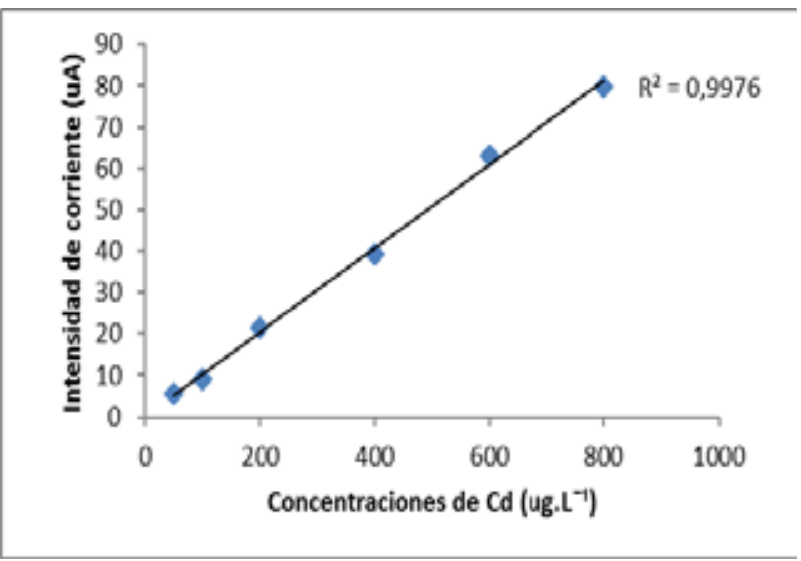

Fuente: Elaboración propia

Figura 6. Curva de calibración de cadmio por adición de soluciones estándar de 50, 100, 200, $400,600,800 \mu \mathrm{g} . \mathrm{L}^{-1}$

\section{Límite de detección, límite de cuantificación y precisión}

Los límites de detección y cuantificación se obtuvieron a través de varias mediciones de corriente mediante un blanco analítico a un potencial de $-0,7 \mathrm{~V}$, lo que permitió obtener un límite de detección de $0,1168 \mu \mathrm{g} \cdot \mathrm{mL}^{-1} \mathrm{y}$ un límite de cuantificación de $0,3549 \mu \mathrm{g} \cdot \mathrm{mL}^{-1}$ para el método de barrido lineal, y un límite de detección de $1,70 \mu \mathrm{g} \cdot \mathrm{L}^{-1} \mathrm{y}$ límite de cuantificación de $5,17 \mu \mathrm{g} . \mathrm{L}^{-1}$ para el método de onda cuadrada. Asimismo, se determinó el coeficiente de variación de 4 muestras para 5 niveles de concentración $\left(2-10 \mu \mathrm{g} \cdot \mathrm{mL}^{-1}\right.$ y $\left.50-800 \mu \mathrm{g} . \mathrm{L}^{-1}\right)$ a partir de estos datos se determinó la desviación estándar relativa, que según la FDA (Food and Drug Administration) (2001) debe ser menor al $20 \%$ para concentraciones de nivel bajo y menor del $15 \%$ para concentraciones medias y altas ${ }^{15}$. Es así que este parámetro analítico fue de 7,5 \% y $6,5 \%$, lo que nos permite concluir que sí cumple con los parámetros establecidos de validación analítica de métodos según la FDA (Food ang Drug Administration) (2001).

\section{CONCLUSIONES}

La voltamperometría de redisolución anódica (ASV) nos permitió obtener resultados confiables, con la modificación del electrodo de carbón vítreo con película de bismuto in situ (BiFE) con límites de detección aceptables y una amplia ventana de potencial negativo. Se obtuvieron voltamogramas bien definidos, los cuales mostraron un incremento de la corriente al aumentar la concentración del analito. Con estos datos se realizaron las curvas de calibración que resultaron lineales para los sistemas de voltamperometría de barrido lineal 
y onda cuadrada con coeficientes de correlación de 0,$997 ; 0,992 ; 0,998$, respectivamente. También se obtuvieron límites de detección de $0,1168 \mu \mathrm{g} \cdot \mathrm{mL}^{-1}$ y $1,70 \mu \mathrm{g} \cdot \mathrm{L}^{-1}$ y límite de cuantificación de $0,3549 \mu \mathrm{g} \cdot \mathrm{mL}^{-1}$ y $5,17 \mu \mathrm{g} \cdot \mathrm{L}^{-1}$ para los métodos electroanalíticos antes mencionados. Adicionalmente, la precisión de los métodos se vio reflejada en la desviación estándar relativa con valores de 7,5\% y 6,5\%, lo que nos dio la certeza de que las técnicas son confiables para la medición de trazas de este metal. Comparando las dos técnicas electroanalíticas, la voltameperometría de onda cuadrada resultó ser más sensible, con una mayor linealidad, así como también, mayor rapidez para la determinación de cadmio.

\section{AGRADECIMIENTO}

Los autores agradecen al grupo y semillero de investigaciones Aplicaciones electroanalíticas de la carrera de Ingeniería Química de la Universidad Técnica de Machala por el apoyo brindado.

\section{REFRERENCIAS BIBLIOGRÁFICAS}

1. Acosta I, Moctezuma-Zárate M de G, Cárdenas JF, Gutiérrez C. Bioadsorción de Cadmio ( II ) en Solución Acuosa por Biomasas Fúngicas. Inf Tecnol. 2007;18(1):9-14.

2. Li J, Guo S, Zhai Y, Wang E. Nafion - graphene nanocomposite film as enhanced sensing platform for ultrasensitive determination of cadmium. Electrochem Commun. 2009;11(5):1085-1088.

3. Suciu P, Vega M, Roman L. Determination of cadmium by differential pulse adsorptive stripping voltammetry. J Pharm Biomed Anal. 2000;23:99-106.

4. Rutyna I, Korolczuk M. Determination of lead and cadmium by anodic stripping voltammetry at bismuth film electrodes following double deposition and stripping steps. Electroanalysis. 2014;26(12):136-141.

5. Ouyang R, Zhu Z, Tatum CE, Chambers JQ, Xue Z-L. Simultaneous stripping detection of $\mathrm{Zn}$ ( II ), Cd ( II ) and $\mathrm{Pb}$ ( II ) using a bimetallic $\mathrm{Hg}-\mathrm{Bi} /$ single-walled carbon nanotubes composite electrode. J Electroanal Chem. 2011;656:78-84.

6. Zhu L, Xu L, Huang B, Jia N, Tan L, Yao S. Simultaneous determination of Cd ( II ) and $\mathrm{Pb}$ ( II ) using square wave anodic stripping voltammetry at a gold nanoparticlegraphene- cysteine composite modified bismuth film electrode. Electrochim Acta. 2014;115:471-477.

7. Ashrafi AM, Vytřas K. Codeposited Antimony-Bismuth Film Carbon Paste Electrodes for Electrochemical Stripping Determination of Trace Heavy Metals. Int J Electrochem Sci. 2013;8:2095-2103.

8. Roohollah K, Craig B, Xiaobo J, Richard C. Electroanalytical Determination of Cadmium ( II ) and Lead ( II ) Using an in-situ Bismuth Film Modified Edge Plane Pyrolytic Graphite Electrode. Anal Sci. 2007;23:283-289. 
9. Ho G, Kyu W, Shik J, Goon S. Determination of trace metals by anodic stripping voltammetry using a bismuth-modified carbon nanotube electrode. Talanta. 2008;76:301-308.

10. Carvalho LM de, Nascimento PC do, Koschinsky A, Bau M, Stefanello RF. Simultaneous Determination of Cadmium , Lead, Copper, and Thallium in Highly Saline Samples by Anodic Stripping Voltammetry ( ASV ) Using Mercury-Film and Bismuth-Film Electrodes. Electroanalysis. 2007;19(16):1719-1726.

11. Demetriades D, Economou A, Voulgaropoulos A. A study of pencil-lead bismuth-film electrodes for the determination of trace metals by anodic stripping voltammetry. Anal Chim Acta. 2004;519:167-172.

12. Bonfil Y, Kirowa E. "Determination of nanomolar concentrations of lead and cadmium by anodic-stripping voltammetry at the silver electrode". Anal Chim Acta. 2002;457:285296.

13. Ortiz D, Betancourt H. Aplicación de técnicas electroanalíticas en la determinación de cadmio en agua potable. [Internet]. Machala: Universidad Técnica de Machala; 2015. [Citado el 22 jul 2016]. Disponible en: http://repositorio.utmachala.edu.ec/ bitstream/48000/3648/1/CD000025-TRABAJO\%20COMPLETO-pdf

14. Kokkinos C, Economou A, Raptis I, Efstathiou CE. Lithographically fabricated disposable bismuth-film electrodes for the trace determination of $\mathrm{Pb}$ ( II ) and $\mathrm{Cd}$ ( II ) by anodic stripping voltammetry. Electrochim Acta. 2008;53:5294-5299.

15. Ramirez C, Olarte E, Tellez E, Palma M. Validación del método analítico para la determinación de mercurio total en sangre humana por espectrofotometría de absorción atómica Zeeman RA-915 + con el módulo de pirólisis PYRO-915 +. Rev Colomb Quim. 2013;42(3):11-66. 